Article DOI: https://doi.org/10.35219/ann-ugal-math-phys-mec.2019.1.14

\title{
ANTIBACTERIAL PROPERTIES OF SILVER NANOPARTICLES DEPOSITIONS ON CELLULOSE NANOFIBERS SURFACES USING REDUCING AGENTS
}

\author{
Radu Costin Pană ${ }^{1}$ \\ ${ }^{1}$ University of Bucharest, Faculty of Physics, Bucharest, Romania, email: radu.pana@gmail.com
}

\begin{abstract}
Polymer cellulose nanofibers have been heavely utilized in the biomedical industry in order to create and produce artificial tissue, bio-sensing devices and biocompatible wearable materials. Because cellulose itself presents important characteristics for medical applications along the lines of biodegradability and biocompatibility (i.e. it makes an ideal component for temporary anchorage devices and mini-implants fixed to bones) and due to the fact that the cellulose does not natively presents antibacterial properties, an antibacterial cellulose based nanocomposite was conceived by depositions of silver nanoparticles (AgNPs) on cellulose nanofibers. In order to examine the properties of the newly created nanomaterial a scanning electron microscope and a Xray diffraction detector were used. The examinations have been conducted in regards to confirm that the spectra of the resultant nanocomposite presents strong broad similarities to silver compounds being known to have strong antibacterial characteristics. The antibacterial test of the composite was confirmed using tests against the Gram-negative bacteria (E.Coli) and as well Gram-positive bacteria (S. aureus). The samples have shown bactericidal activities and bacterial inhibition efficiencies. Silver nanoparticles on cellulose nanofibers display distinguished antibacterial features for the purpose of medical applications among others utilizations. In this study we present the bacterial growth inhibition properties of cellulose nanofibers silver nanoparticles nanocomposite.
\end{abstract}

Keywords: antibacterial cellulose, nanofibers, nanocomposites, silver nanoparticles.

\section{INTRODUCTION}

Nanocomposites generated from silver polymers or AgNPs have been utilized up until now in a multitude of fields such as in biomedical, conductive, catalysis and optical applications. These nanocomposites have already shown certains properties of the polymer used and desirable properties of AgNPs. Because of increasing awareness about environmental protection, biocompatible and biodegradable polymers such as cellulose have in present time great significance.

Cellulose can have certain specific properties indispensable for numerous medical applications such as very good hydrophilicity, biodegradability and biocompatibility special characteristics. In particular for antibacterial utilization, the cellulose can effectively guarantee specifications needed. Silver and its nanoparticles are well-known for their special uses in bactericidal, electric, thermal, optical and catalytic applications.

Other applications of silver nanoparticles have been used in the purification of water, treatments of wounds, cuts, burns and as disinfectant, being an excellent antibacterial and antifungal 
agent. It is toxic to viruses, efficient against microorganisms that are resistant to certain antibiotics and have already proven effectiveness against cancer cells. As well, gradual release of silver ions, AgNPs have proven special properties in bacterial growth inhibition and bactericidal properties. Silver nanoparticles rupture can change as well organisms DNA and thus effectively killing the bacteria in organisms [1].

However, well dispersed AgNPs in the cellulose matrix are required because otherwise the antimicrobial effect can steeply decrease. Nevertheless, other parameters that are important, such as distributions of particle size, cationic silver release, metal content and interaction with the surface of cellulose can greatly influence the antimicrobial activity of these nanocomposites [2].

Because of all important characteristics previously presented (i.e. it makes an ideal component for temporary anchorage devices and mini-implants fixed to bones) and due to the fact that the cellulose does not natively presents antibacterial properties, an antibacterial cellulose based nanocomposite was conceived by depositions of silver nanoparticles (AgNPs) on cellulose nanofibers.

\section{NANOCOMPOSITE PREPARATION}

For this paper it was used the in situ approach to prepare a dispersion of NPs in cellulose matrices involves the entrapment of metal cations in the fibers followed by their reduction with an external reducing agent. In this procedure the reducing agent also act as a co-stabilizer (together with the cellulose fibers) for the AgNPs. Sodium borohydride has been used to reduce metal ions in cellulose matrices [3].

In order to examine the properties of the newly created nanomaterial a scanning electron microscope and a X-ray diffraction detector were used. The examinations have been conducted in regards to confirm that the spectra of the resultant nanocomposite presents strong broad similarities to silver compounds being known to have strong antibacterial characteristics. The antibacterial test of the composite was confirmed using tests against the Gram-negative bacteria (E.Coli) and as well Grampositive bacteria ( $S$. aureus) [4].

The samples have shown bactericidal activities and bacterial inhibition efficiencies. Silver nanoparticles on cellulose nanofibers display distinguished antibacterial features for the purpose of medical applications among others utilizations. In this study we present the bacterial growth inhibition properties of cellulose nanofibers silver nanoparticles nanocomposite.

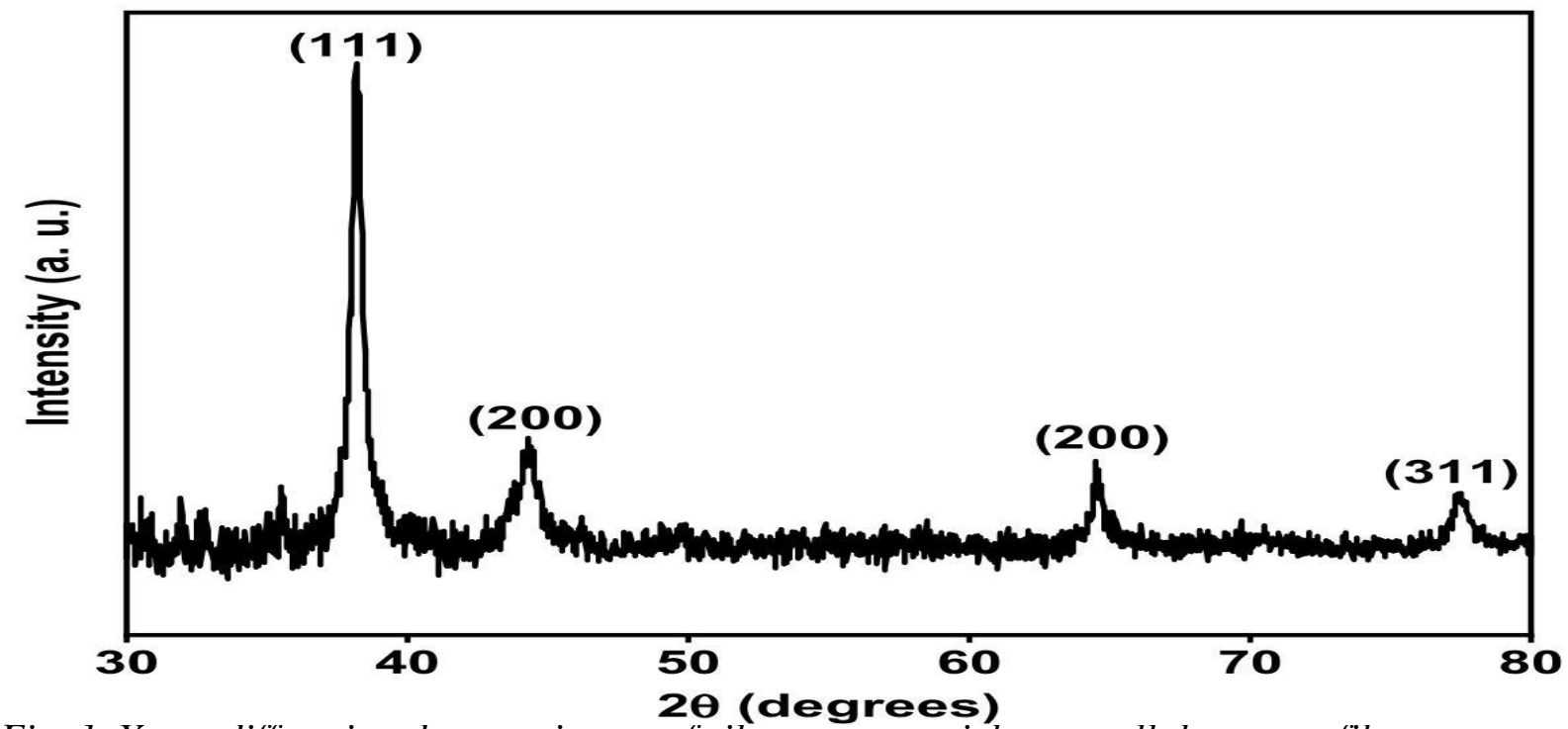

Fig. 1. X-ray diffraction detector image of silver nanoparticles on cellulose nanofibers 

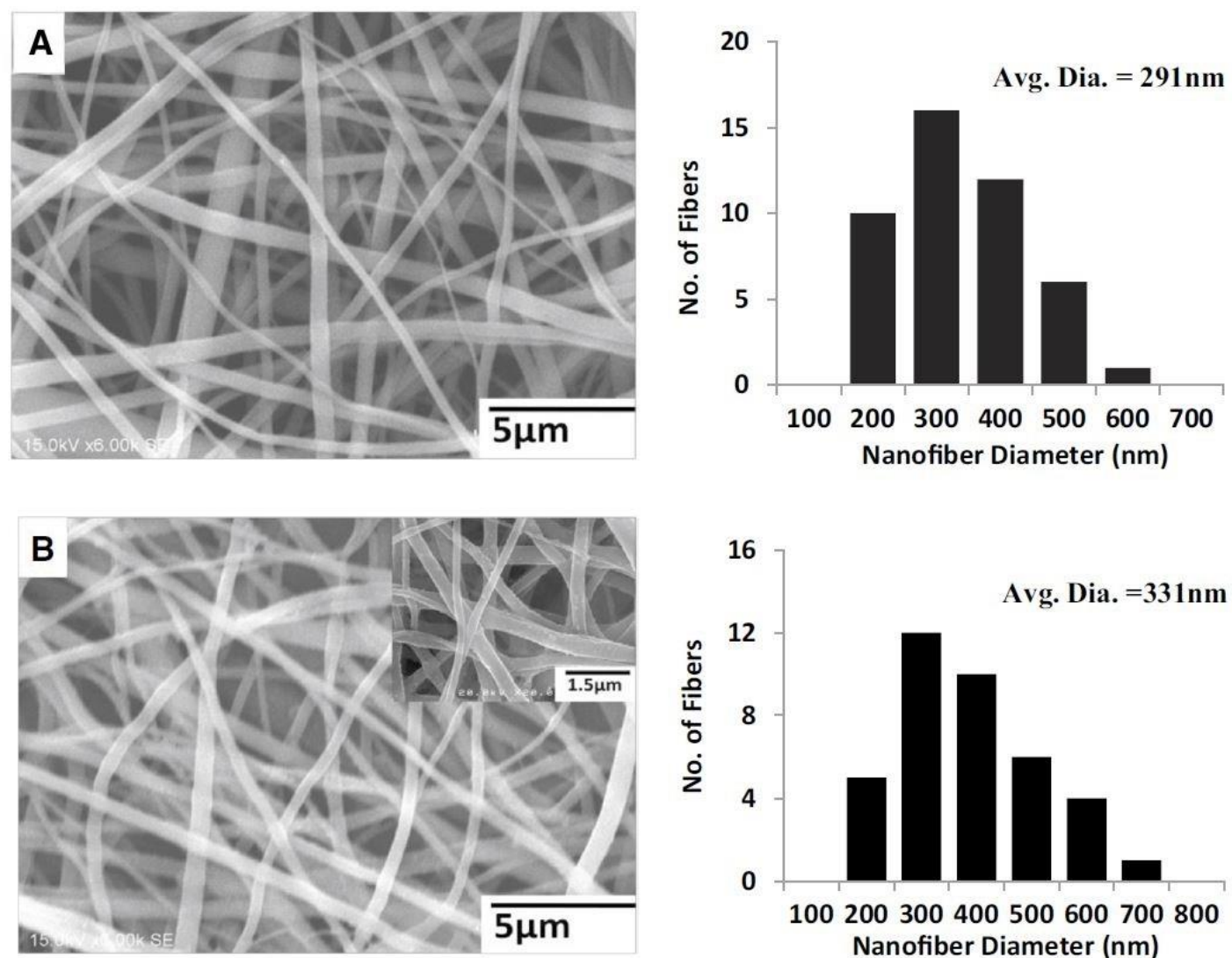

Fig. 2. Scanning electron microscope images of cellulose nanofibers $(A)$ and silver nanoparticles on cellulose nanofibers $(B)$

\section{METHODS OF INVESTIGATION}

The X-ray diffraction instrument (XRD) was used for X-ray diffraction investigation and a scanning electron microscope (SEM), was utilized to gain information about the morphologies of the samples. The samples have been coated with silver (Ag) for $160 \mathrm{~s}$ at $20 \mathrm{~mA}$ to form $8 \mathrm{~nm}$ conductive coating before SEM observations. The antibacterial properties tests were conducted by obtaining E. coli and S. aureus strains using serial dilutions of the overnight culture. The samples were added into $5^{-4} \mathrm{~L}$ of culture using $48 \mathrm{~h}$-well cell culture plates and incubated at $37{ }^{\circ} \mathrm{C}$. At specific time intervals (every hour), the absorbance values were taken.

The quantitative bactericidal performance of the samples was analyzed by counting colonyforming units and plotting the results as cell viability relative to control (colony-forming units (CFU)). For both the test strains (E. coli and S. aureus), the CFU/mL was adjusted to dilutions of the culture. Then, $1^{-4} \mathrm{~L}$ of the culture interacted with the samples (contacted cells) and without samples were spread on agar plates and incubated over a period of 12 hours at $37^{\circ} \mathrm{C}$.

\section{RESULTS}

The X-ray diffraction (XRD) analysis (Fig. 1) of silver nanoparticles on cellulose nanofibers (Fig. 2B) shown formation of metallic silver nanoparticles. The diffraction angles at $37.5^{\circ}, 46.2^{\circ}$, $63.75^{\circ}$ and $76.1^{\circ}$ are indexed as (111), (200), (220) and (311) planes of silver metallic structure; thus confirming the fact that the silver nanoparticles are pure and in crystalline state.

The SEM images of the samples shown in Fig. 2 are characteristic for regular and smooth cellulose nanofibers and silver nanoparticles on cellulose nanofibers. The average size of cellulose nanofibers was found to be $291 \mathrm{~nm}$ and respectively $331 \mathrm{~nm}$ for silver nanoparticles on cellulose nanofibers. Increase in the average diameters of the silver nanoparticles on cellulose nanofibers can be 
attributed to the formation of newly created coating on nanofiber surfaces.

\section{CONCLUSIONS}

The combination of metal nanoparticles and distinct types of cellulose matrices takes benefit of the properties of both components and simultaneously might result in properties due to synergistic effects. Besides the nature of the components in the final nanocomposite, the performance of the final material depends on the preparative methodologies employed in their production. The combination of silver nanoparticles in cellulosic matrices is an important but less exploited strategy to prepare multifunctional composites.

\section{References}

1. Kong H., Jang J., Antibacterial properties of novel poly (methyl methacrylate) nanofiber containing silver nanoparticles, Langmuir, 24(5), pp. 2051-2056, 2008.

2. Martins N. C. T., Freire C. S. R., Pinto R. J. B., Fernandes S. C. M., Neto C. P., Silvestre A. J. D., Causio J., Baldi G., Sadocco P., Trindade T., Electrostatic assembly of Ag nanoparticles onto nanofibrillated cellulose for antibacterial paper products, Cellulose 19(4), pp. 1425-1436, 2012.

3. Balazs A. C., Emrick T., Russell T. P., Nanoparticle polymer composites: Where two small worlds meet, Science, 314(5802), pp. 1107-1110, 2006.

4. Zhang F. J., Chen M. L., Oh W. C., Photoelectrocatalytic propertiesand bactericidal activities of silver-treated carbon nanotube/titania composites. Compos. Sci. Technol., 71(5), pp. 658$665,2011$. 